\title{
TM8 represses developmental timing in Nicotiana benthamiana and has functionally diversified in angiosperms
}

\author{
Heleen Coenen ${ }^{1 \dagger}$, Tom Viaene ${ }^{1 \dagger}$, Michiel Vandenbussche ${ }^{2}$ and Koen Geuten ${ }^{1 *}$
}

\begin{abstract}
Background: MADS-box genes are key regulators of plant reproductive development and members of most lineages of this gene family have been extensively studied. However, the function and diversification of the ancient TM8 lineage remains elusive to date. The available data suggest a possible function in flower development in tomato and fast evolution through numerous gene loss events in flowering plants.

Results: We show the broad conservation of TM8 within angiosperms and find that in contrast to other MADS-box gene lineages, no gene duplicates have been retained after major whole genome duplication events. Through knock-down of NbTM8 by virus induced gene silencing in Nicotiana benthamiana, we show that NbTM8 represses miR172 together with another MADS-box gene, SHORT VEGETATIVE PHASE (NbSVP). In the closely related species Petunia hybrida, PhTM8 is not expressed under the conditions we investigated and consistent with this, a knock-out mutant did not show a phenotype. Finally, we generated transgenic tomato plants in which TM8 was silenced or ectopically expressed, but these plants did not display a clear phenotype. Therefore, no clear function could be confirmed for Solanum lycopersium.
\end{abstract}

Conclusions: While the presence of TM8 is generally conserved, it remains difficult to propose a general function in angiosperms. Based on all the available data to date, supplemented with our own results, TM8 function seems to have diversified quickly throughout angiosperms and acts as repressor of miR172 in Nicotiana benthamiana, together with NbSVP.

Keywords: TM8, SVP, VIGS, Nicotiana benthamiana, Repressor of miR172, RNAi, Overexpression, Solanum lycopersium, Petunia hybrida

\section{Background}

MIKC ${ }^{\mathrm{C}}$-type MADS-box genes are plant transcription factors involved in diverse developmental processes [13]. In gymnosperms, 12 clades can be distinguished while 17 clades are present in angiosperms as a result of duplication events [4]. MICK $^{\mathrm{C}}$-type MADS-box genes are generally known for their roles in the flowering transition, floral meristem and floral organ identity, fruit and seed development. Their key role in plant reproductive processes, often of interest for crop improvement, has led to the functional characterization of most members

\footnotetext{
* Correspondence: koen.geuten@kuleuven.be

${ }^{\dagger}$ Heleen Coenen and Tom Viaene contributed equally to this work.

'Department of Biology, KU Leuven, Kasteelpark Arenberg 31, B-3001 Leuven, Belgium

Full list of author information is available at the end of the article
}

in at least one species. The function of a few MIKC ${ }^{\mathrm{C}}$ type lineages, however, remains elusive, and the Tomato MADS 8-clade (TM8) is one of these [5].

Because of its absence in classical model species such as Arabidopsis, maize and rice, TM8 was originally not functionally characterized in any species. A recent phylogenomic study evaluating the evolutionary conservation of MICK-type MADS box genes in angiosperms, describes five independent losses of the TM8-clade throughout the angiosperms, making it the most often lost MADS-box lineage, next to FLOWERING LOCUS C $(F L C)$ [6]. On the other hand, as a result of the increasing number of available genomes and sequences, it became clear that TM8 homologs appear to be present throughout the spermatophytes.

(c) The Author(s). 2018 Open Access This article is distributed under the terms of the Creative Commons Attribution 4.0 International License (http://creativecommons.org/licenses/by/4.0/), which permits unrestricted use, distribution, and 
Tomato MADS 8 (TM8) was the first member of the clade to be identified when it was isolated from Solanum lycopersicum. It was classified as an 'early' flowering gene, expressed in floral meristems and to a lesser extent in the three inner floral whorls [7]. Its first characterization was also in tomato. Overexpression of full length TM8-antisense RNA resulted in defects of the female floral organs, male and female sterility and parthenocarpy in three out of 12 transgenic lines [8]. More recently, in an effort to thoroughly characterize $T M 8$, Daminato et al. produced transgenic tomatoes overexpressing full-length TM8 and plants expressing TM8 fused to the SRDX transcriptional repressor domain [9]. They observed malformations of stamens in three out of 22 independent TM8 overexpression lines, and consistent with this, expression of B-genes was altered in these flowers. The 15 35S:TM8:SRDX lines displayed more pronounced phenotypes like epinastic darker green leaves and oblong ovaries and fruits, which were seedless. Flower and fruit peduncles appeared longer and the abscission zone was abnormal. JOINTLESS, an SVP homolog, was significantly down regulated in these plants. The authors concluded that TM8 plays some role in the development of tomato flowers by possible interactions with MACROCALYX.

Aside from this study, hardly any functional data on TM8 have been presented thus far. Few studies, mostly pursuing a genome wide analysis of MADS-box genes in a certain species, report the presence of TM8 in a diversity of genomes and a function has sometimes been proposed based on gene expression data. In Cucumis sativus, ERAF17, the cucumber TM8 ortholog, is involved in the development of female flowers after induction by ethylene [10]. In Antirrhinum majus, the TM8 ortholog $D E F H 7$, is only expressed in young bracts (unpublished observations, [11]). Hileman et al. detected TM8 in nearly all tomato tissues, albeit to a lesser extent in roots, seedlings, carpels and green fruit [12]. In Vitis vinifera, $V v T M 8$ was detected in latent buds during the flowering transition, in later stages of flower development and in tendrils [13]. However, in a more recent transcriptional analysis of tendril and inflorescence development, VvTM8 was not mentioned [14]. In a molecular study of fleshy fruit-like structures in gymnosperms, TM8 was unexpectedly identified [15], again displaying a broad expression pattern in both vegetative and reproductive structures. TbTM8 was most highly expressed during arilus development in Taxus baccata. In Ginkgo biloba, three TM8-like genes are expressed in leaves, but are also present in male and female cones and during seed and fleshy sarcotesta development. Based on these observations, TM8 was assigned a role in fleshy fruit-like development in gymnosperms. In another genome-wide identification of MADS-box genes in Prunus mume, PmMADS26 was identified as the sole TM8 ortholog expressed only in the pistil and the fruit, its expression gradually increasing during ripening [16]. In the closely related Prunus persica on the other hand, the TM8 ortholog, identified as Ppe$M A D S 35$, was only very lowly expressed in roots, leaves, cotyledons, embryo's and fruits [17]. In a study in pear, PpTM8-1 showed high expression in the bud, branch, leaf and root but not in the flower, while $P p T M 8-2$ was highly expressed in all tissues [18]. A similar pattern was found in Malus domesticus, where one TM8 homolog, MdMADS045 showed expression in fruits and strong expression during flower development, while the other, MdMADS111, was rather lowly expressed in most tissues except in the developing fruits [19].

Though no clear general or conserved role for TM8 can be concluded from these diverse expression data, most studies share a broad TM8 expression pattern, both in vegetative and reproductive tissues and most often during floral meristem and fruit development. Therefore, TM8 is most commonly assigned a function in flower and fruit development [9, 13, 15]. However, clear knock-down or knock-out mutants are not available and seem necessary to provide more insight into its true role. In this study, we evaluate the function of TM8 function within Solanaceae by means of a Petunia hybrida (Petunia) PhTM8 knock-out mutant, and transgenically altered TM8 expression in Nicotiana benthamiana (Nicotiana) and Solanum lycopersicum (tomato). The diverse results obtained in this work combined with the miscellaneous data from previous studies listed above, support the idea of rapid functional evolution of the TM8 gene.

\section{Results}

TM8 was not retained after major genome duplication events

Recent studies showed that TM8 is highly conserved in gymnosperms, and that this clade even expanded in gymnosperms, becoming one of the largest MADS-box clades in fruitless seed plants [4]. In angiosperms on the other hand, it is designated to be the most often lost MADS-box subclade together with FLC [6]. To gain further insight in its evolutionary history we reconstructed its phylogeny in angiosperms, thereby not only focussing on where it was lost, but also trying to describe its widespread conservation. To do this, we used sequences from all available genomes to date in Phytozome 12 and the Sol Genomics Network (Additional file 1), supplemented with sequences from EST and transcriptome databases on NCBI and the oneKP platform [20, 21]. Special efforts were made to identify orthologs in species belonging to orders lacking fully available genomes. 
The resulting phylogeny clearly demonstrates that TM8 is indeed present in many orders throughout the angiosperms and that the gene phylogeny follows the angiosperm phylogeny (Fig. 1, Additional file 2). This implies that no major duplications occurred within the TM8-lineage, which is in contrast to most other MADS-box genes which, like other transcription factors, are generally conserved after whole genome duplications $[22,23]$. On the other hand, the number of cases in which TM8 was lost or could not be identified are strikingly numerous and well spread throughout angiosperm evolution. No TM8 orthologs could be detected in species belonging to Nymphaeales, to all monocots minus the Alismatales, to Trochodendrales, to Gunnerales, Dilleniales, Santanales, Fabales, Gentianales or Boraginales in addition to other smaller orders and many important families like the Brassicaceae. Only one or a few orthologs were identified in the Chloranthales, Proteales, Buxales, Geraniales and Cornales. While we cannot draw final conclusions about its loss in a species when no fully sequenced genome is available, to date many species are broadly sampled and have extensive transcriptome data available. The lack of TM8 from these databases does suggest its absence in these taxa.

\section{NbTM8 and NbSVP repress miR172 in Nicotiana benthamiana}

We decided to use Nicotiana benthamiana for the initial characterization of TM8, as it has been proven to be an excellent model species for virus induced gene silencing (VIGS), a rapid and easy method to obtain loss of function phenotypes [24]. To first evaluate when and where TM8 functions in Nicotiana, we used qPCR and in situ hybridization (Fig. 2). The qPCR results show that NbTM8 is strongly expressed in the stem and to a lesser extent in seedlings, leaves and floral organs (Fig. 2a). The in situ hybridizations reveal NbTM8 expression in both developing shoots and flowers (Fig. 2b, c; Additional file 3). Expression in a developmental series of plants, each with one more leaf, shows a strong peak early in vegetative development around the initiation of the fourth leaf. Its expression drops after this peak and increases again only around the ninth or tenth leaf and continues to increase until the first flower appears (14th leaf) (Fig. 2d). We hypothesized that the first peak might be around the juvenile-adult transition, therefore we also quantified miR156 and miR172 levels by stem-loop qPCR (Fig. 2e). In agreement with this hypothesis we found that the first peak in NbTM8 expression coincides almost perfectly with the moment that miR156 drops below the increasing miR172 level. In Arabidopsis, this juvenile-adult transition is characterized by the production of trichomes on the abaxial sides of leaves [25], so we studied the juvenile-adult transition in Nicotiana to identify similar markers for this phase change. We observed that both large trichomes and a pointed leaf tip emerge around the third and fourth leaf, confirming that the phase transition indeed takes place at this point (Additional file 4A-C). The second peak in NbTM8 expression coincides with the increased expression of a homolog of SUPPRESSOR OF CONSTANS 1 in Nicotiana (NbSOC1) as shown in the graph by a grey arrow (Fig. $2 \mathrm{~d}$, e and Additional file 4D). SOC1 is a marker of floral induction in Arabidopsis and is strongly expressed during the floral transition [26]. These observations suggest that NbTM8 acts during both phase transitions in Nicotiana, possibly by regulating miR156 and $m i R 172$.

As MADS-box genes often function in heteromeric complexes, we considered whether a protein interaction partner might be involved in this regulation. A good candidate would be SHORT VEGETATIVE PHASE $(S V P)$, another MADS-box gene involved in the floral transition by down regulation of miR172 in Arabidopsis [27]. We first tested the ability of NbSVP and NbTM8 to interact using yeast-two-hybrid. While we found a positive interaction in one direction (AD-SVP + BD-TM8), the result in the other direction (AD-TM8 + BD-SVP) remains inconclusive due to the auto-activation of NbSVP (Fig. 2f). Therefore, we subsequently performed co-immunoprecipitation from Arabidopsis leaf mesophyll protoplasts and confirmed that these proteins can interact in vitro (Fig. $2 \mathrm{~g}$ ).

Therefore, we decided to also characterize $N b S V P$. qPCR reveals that $N b S V P$ is more strongly expressed in seedlings and leaves and to a lesser extent in the stem when compared to NbTM8 (Fig. 2h). It seems thus that both NbTM 8 and NbSVP acquire their strongest expression levels in the vegetative parts of Nicotiana benthami$a n a$, and that the relative expression of $N b S V P$ in flowers is also lower than for NbTM8 compared to the expression in the vegetative parts. In situ hybridization of $N b S V P$ further reveals that $N b S V P$ is expressed in developing shoots, similar to $N b T M 8$, but in contrast, its expression is absent in floral meristems (Fig. 2i, j; Additional file 3). This is similar to Arabidopsis where SVP expression is constant during vegetative growth but reduces in the inflorescences and further disappears during flower development $[28,29]$. qPCR in a developmental series reveals that in contrast to NbTM8, expression of NbSVP is continuously stronger and shows no major peaks early in vegetative development. Similar to NbTM8, NbSVP mRNA expression increases when the flowering process starts, around the time when Nicotiana APETALA1 (NbAP1) is expressed (Fig. 2k, Additional file 4D).

These data on the co-expression and interaction of NbSVP and NbTM8, suggests that NbTM8 together with NbSVP may have overlapping roles in the transition of the shoot to flowering. Expression of NbTM8 early in 


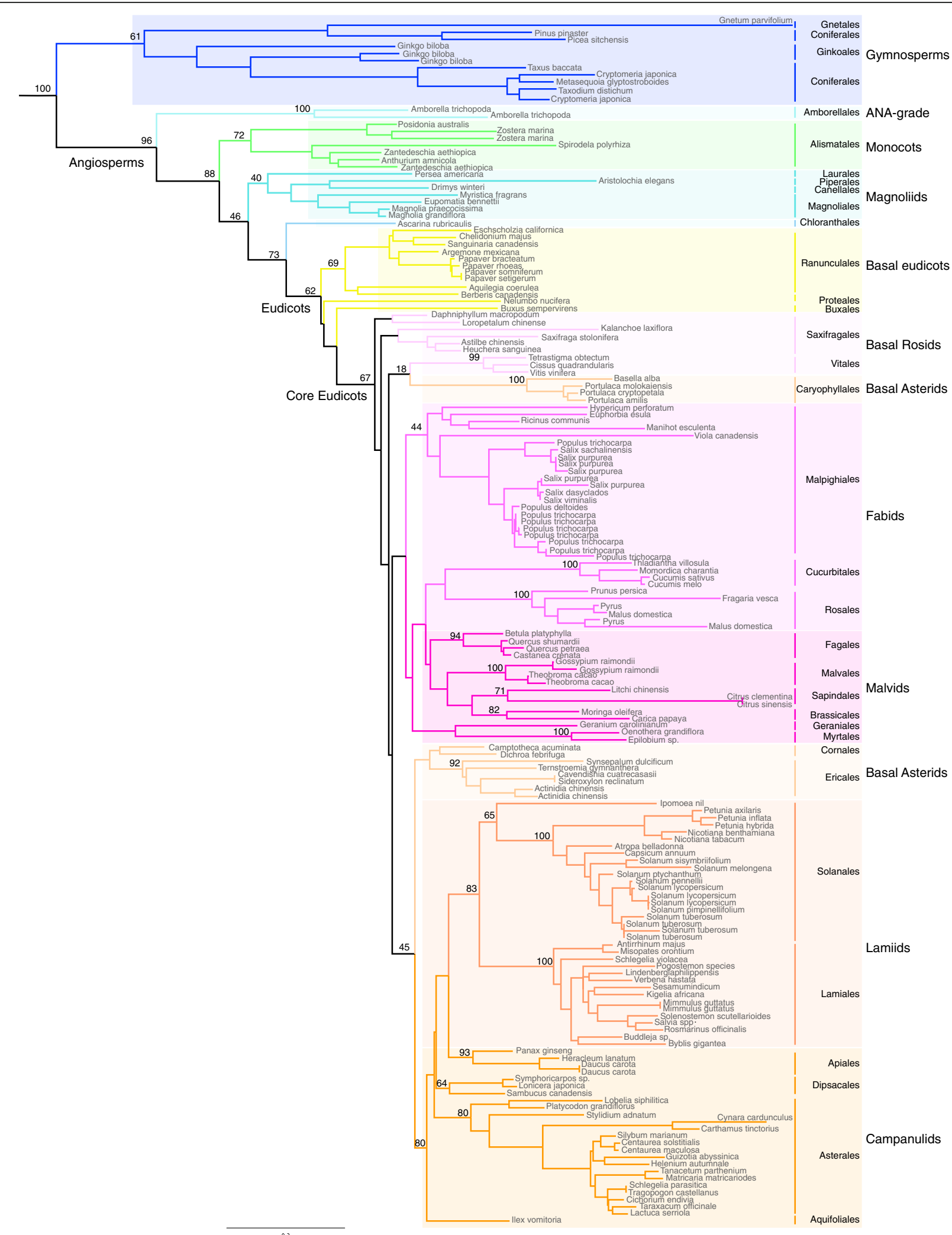

Fig. 1 Maximum Likelihood phylogeny of the TM8-family in angiosperms with bootstrap support. BS support values are shown at major nodes when $>50$. SOC1 genes were used as outgroup (not shown here). In angiosperm orders lacking here, no TM8 orthologs could be identified from the databanks listed in the Methods. Complete phylogeny including all accession numbers is provided as Additional file 2 


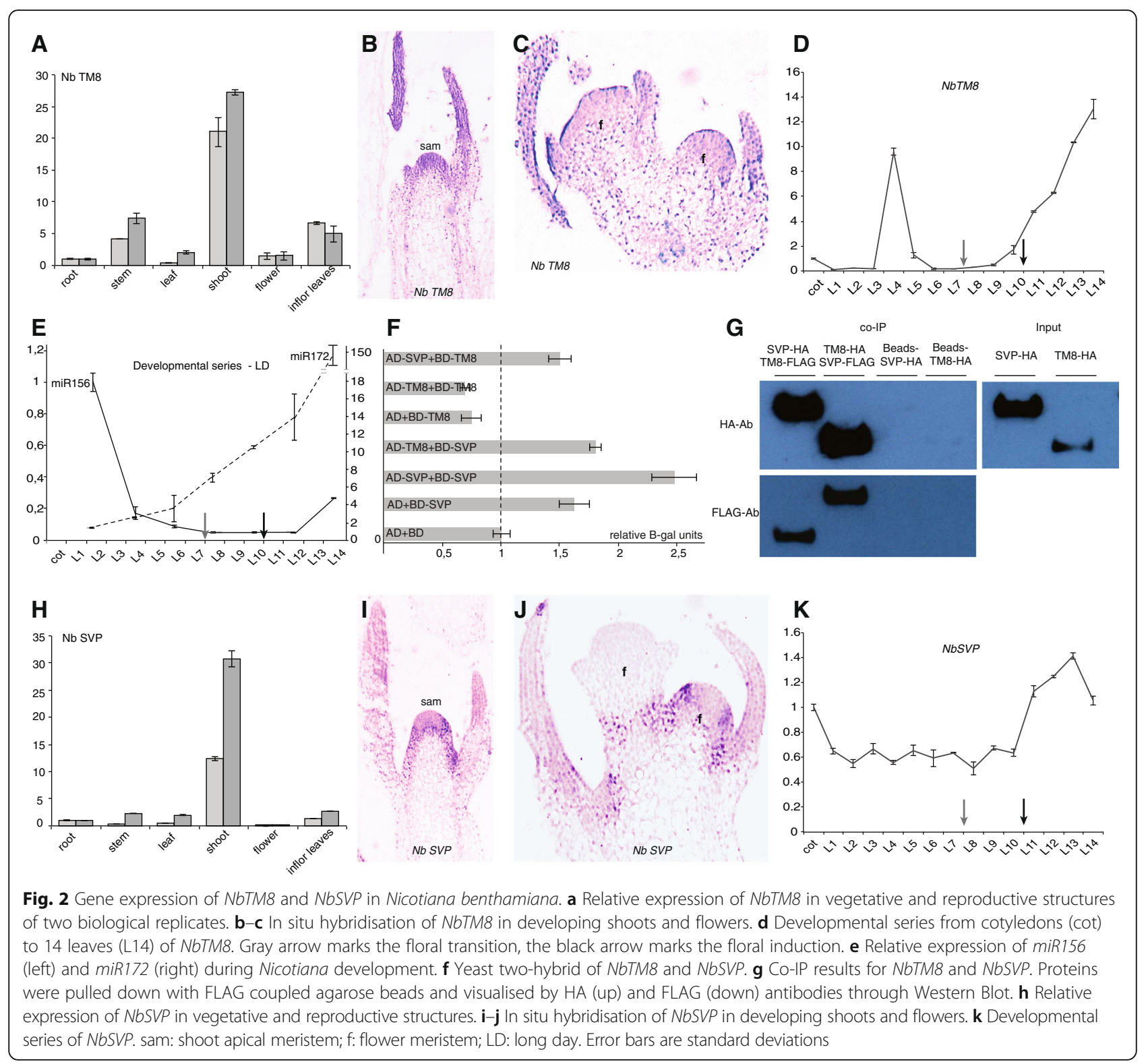

vegetative development further suggests that $N b T M 8$ might play a role in the juvenile to adult transition.

We evaluated the effect of NbTM8 and NbSVP knock down by using virus induced gene silencing. Empty vector (EV) transformed Agrobacterium was injected in control plants. The resulting NbTM8-VIGS plants and NbSVP-VIGS plants were remarkably similar in several ways. NbTM8-VIGS plants displayed a range of characteristic defects in both flowers and inflorescence leaves. The most often observed anomaly (in 15\% of the VIGS flowers compared to $3 \%$ in $\mathrm{EV}, p=0.018$ ) was an increased number of floral organs in the outer three whorls of VIGS plants compared to EV plants, a characteristic also regularly displayed by $N b S V P$-VIGS plants (19\% of the flowers compared to $3 \%$ in EV, $p=0.004$ )
(Fig. 3a). Furthermore, in some cases, NbTM8-VIGS petal tubes were twisted or sometimes even open (Fig. $3 \mathrm{~b}, \mathrm{c})$. Leaves in the inflorescence were smaller, twisted and darker green (Fig. 3d). Overall, NbTM8-VIGS plants can be described as undergoing accelerated development based on the increased rate of flower and leaf production. Both NbTM8-VIGS and NbSVP-VIGS plants were early flowering when using the number of leaves before flowering as a proxy (Fig. 3e-f). They flower early by approximately three leaves compared to control plants, suggesting that both NbSVP and NbTM8 function as repressors of the floral transition in Nicotiana (Fig. 3e). For NbSVP this should not come as a surprise, as $S V P$-like genes have been shown to act as a repressor of the floral transition in Arabidopsis thaliana [28]. For 

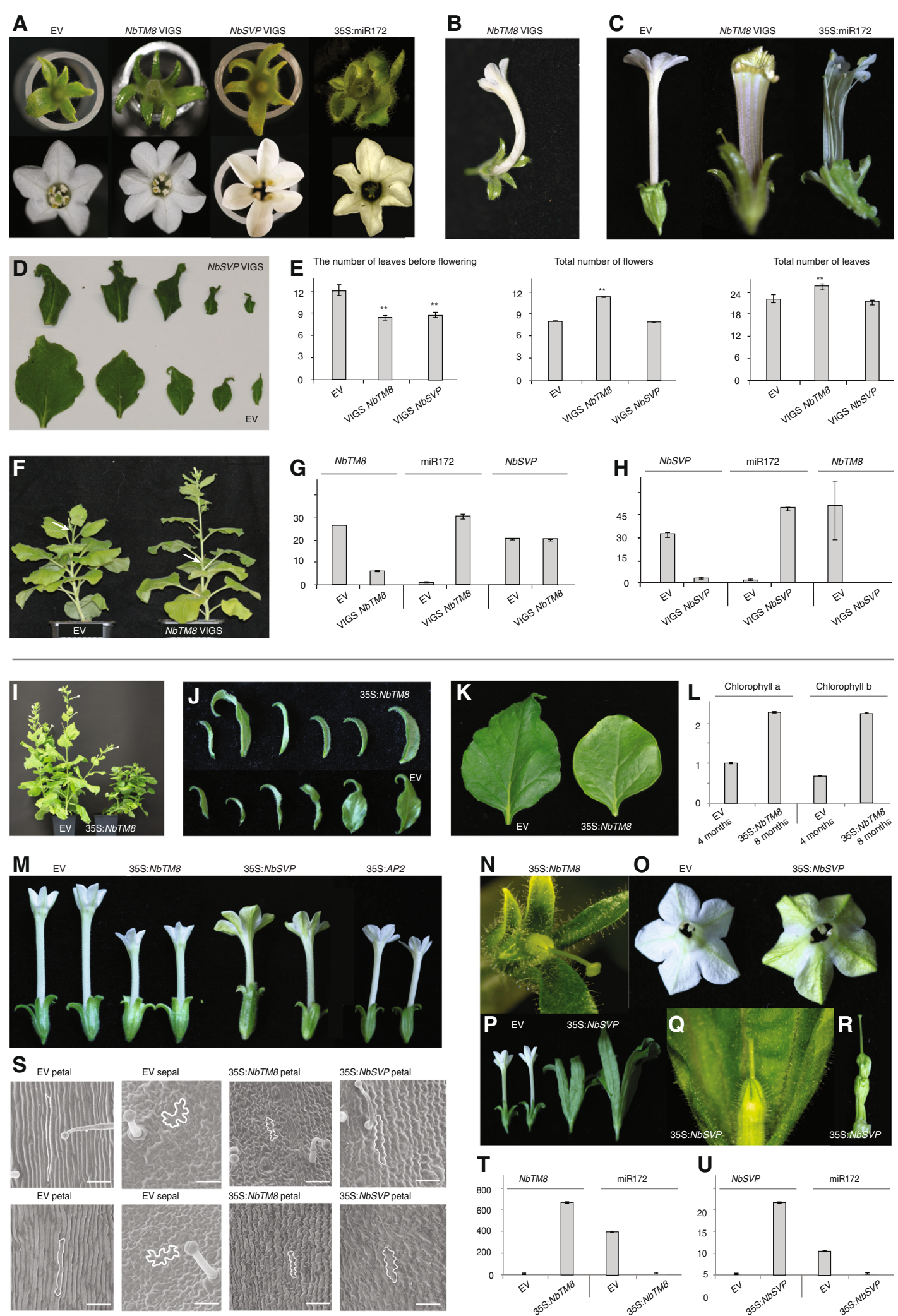

Fig. 3 (See legend on next page.) 


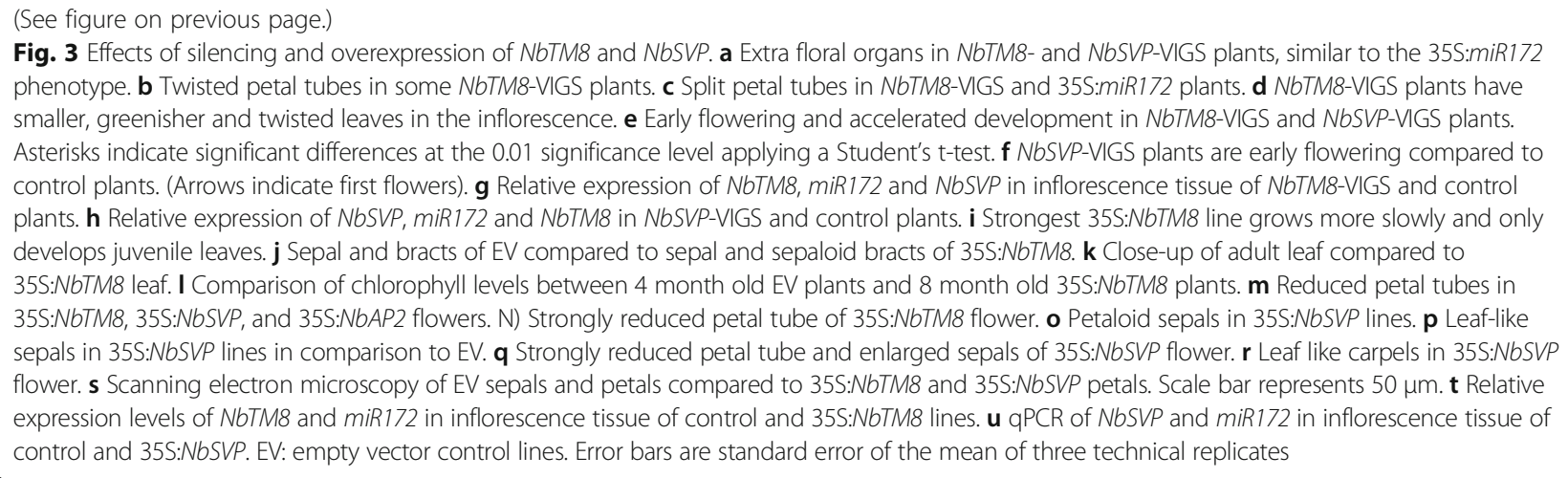

TM8 however, an early flowering phenotype has not yet been attributed to a member of this subfamily. qPCR confirmed much reduced NbTM8 and NbSVP expression in both inflorescence leaves and flowers of NbTM8-VIGS and NbSVP-VIGS plants respectively compared to the EV control (Fig. 3g). Interestingly, the observed phenotypes strongly resemble those of Nicotiana plants that constitutively express Arabidopsis miR172 (Fig. 3a-c) [30]. To verify whether increased expression of miR172 may explain some of the phenotypes, miR172 expression levels were quantified in NbTM8-VIGS and NbSVP-VIGS lines. The results show that miR172 is strongly upregulated in the VIGS lines in comparison to EV control plants (Fig. 3g, h). Because NbTM8 and NbSVP can interact and show similar phenotypes, they might also regulate each other. Indeed, NbSVP silencing results in the concomitant down regulation of NbTM8 (Fig. 3h), while silencing of NbTM8 does not affect the NbSVP level (Fig. 3g).

As silencing seemed to accelerate developmental transitions, we addressed whether constitutive expression would delay these transitions by generating transgenic NbTM8 and NbSVP overexpression plants. While caution is required interpreting overexpression phenotypes due to off-target binding, we observed a number of consistent alterations. Out of all 35S:NbTM8 lines, the strongest one showed a dramatic transformation of all leaves into juvenile leaves and inflorescence bracts remained sepal-like (Fig. 3i, j). Two lines showed a significant increase in lifespan - more than threefold - and senescence was delayed as indicated by a comparison in chlorophyll levels (Fig. 3k, l). Furthermore, these strongest lines did not produce any seed. In all transgenic lines, petal tubes were severely reduced in size and became more greenish (Fig. $3 \mathrm{~m}, \mathrm{n}$ ), a phenotype that was previously observed in plants that constitutively express Arabidopsis APETALA2 (AP2) in Nicotiana [30]. Transgenic plants overexpressing $N b S V P$ also display similar phenotypes as 35S:NbTM8 plants. They also resulted in reduced and greenish petal tubes (Fig. $3 \mathrm{~m}, \mathrm{o}-\mathrm{q})$. Scanning electron microscopy shows that this is a consequence of a partial petal to sepal transformation (Fig. 3s). In other 35S:NbSVP lines the petal tube was even more strongly reduced while sepals were enlarged (Fig. 3p, q). The sepals and carpels were leaf-like, as previously described for ectopically expressed SVP-like genes in other Solanaceae species (Fig. 3p-r) [31]. Both in 35S:NbTM8 and 35S:NbSVP lines, miR172 was significantly down regulated (Fig. 3t, u).

Together, NbTM 8 and NbSVP transcripts have overlapping expression patterns in the shoot apical meristem, in leaves, bracts and in flower meristems. Their expression patterns are uncoupled when sepal primordia emerge and NbSVP expression disappears. Given the above data, it is plausible that NbTM 8 and NbSVP act as negative regulators of miR172 in Nicotiana and may control the timing of developmental transitions together by repressing $m i R 172$ levels.

\section{TM8 appears to have lost its function in Petunia hybrida}

The next step was to evaluate the conservation of this flowering repressor complex in other species. The closely related Petunia is an excellent model, considering the availability of a PhTM8 knock-out mutant which contains a footprint resulting from a $d T p h 1$ transposon insertion in the start codon, leading to a frame shift (Additional file 5). We phenotyped 19 footprint-mutant plants (phtm8) and 21 wild-type plants for developmental and morphological traits, but no differences in flowering time, leaf or flower morphology could be observed (Fig. 4).

To evaluate the effect of PhTM8 overexpression, we tried to generate 35S:PhTM8 lines but we were not able to clone PhTM8 from Petunia cDNA despite of extensive cloning efforts (see methods). In a subsequent search for PhTM8 expression data we found that PhTM8 was also not picked up in a MADS-box gene screening of various Petunia cDNA libraries by Immink et al. [32].

Blasting in all available Petunia transcriptome and EST databases to date did not deliver any hits either, suggesting that PhTM8 is not or only very weakly expressed in 


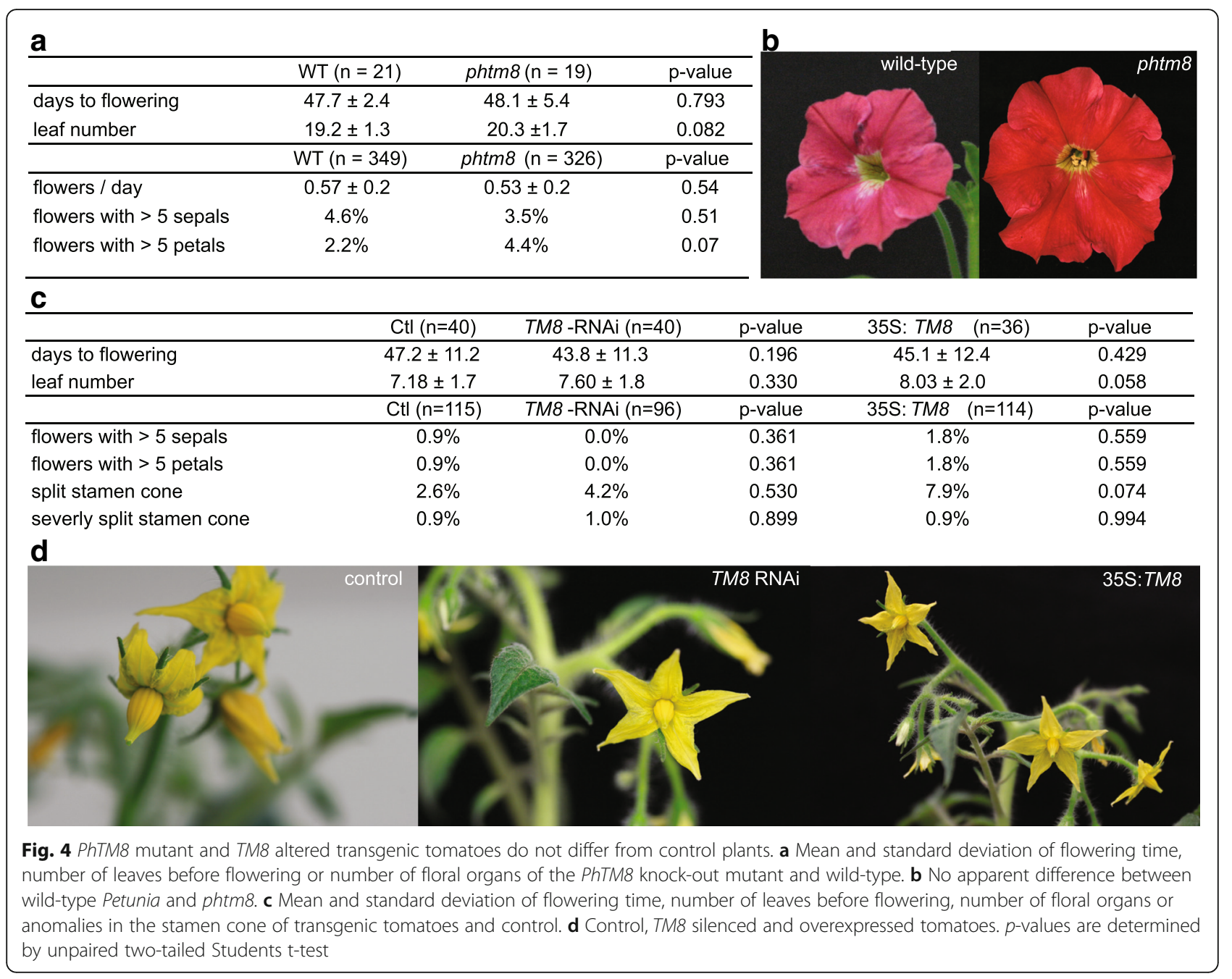

Petunia, at least in normal growth conditions. In the protein sequence however, no obvious deviation from other TM8 orthologs or marks of pseudogenization can be observed (Additional file 6). The protein could therefore still be functional, but might only be expressed in conditions that haven't been tested yet.

Taken together, PhTM8 appears not to act as a floral repressor in Petunia in contrast to NbTM8 in Nicotiana and its function appears to have diverged between these species belonging to the same family.

\section{The function in tomato also remains unclear}

Since Petunia did not seem to express PhTM8, we continued our investigations in tomato, another member of the Solanceae family and the first species in which TM8 was identified [7]. Two overexpression lines and 22 RNAi lines were obtained by Agrobacterium-mediated transformation. Control lines were obtained from uninfected explants grown on non-selective medium. Once transferred to soil, all T0-lines were genotyped and attentively observed for possible abnormalities of vegetative and reproductive organs, but no obvious consistent deviations were observed. Even though many fruits in all three groups were parthenocarpic, enough seed could be obtained from both transgenic and control lines to evaluate TM8 function in the T1 generation, which was not possible using VIGS in Nicotiana. The two 35S:TM8 and 14 selected TM8-RNAi transgenic T1-lines were genotyped and subsequently tested for altered TM8 expression by RT-PCR (Additional file 7).

Based on the results in Nicotiana we expected that altered expression of TM8 could result in a change of flowering time or number of leaves before floral initiation, however, this was not what we observed (Fig. 4c). Only one of the 14 tested TM8-RNAi lines was on average late flowering compared to control, and one 35S:TM8 line was early flowering, opposite to what we observed in Nicotiana, but more important, not 
consistent with any of the other lines. Aside from flowering time, we attentively phenotyped the first three flowers of each T1 plant for deformed stamen cones, extra floral organs and other morphological abnormalities. Again, while the absolute majority of these flowers appeared normal, a few control, TM8-RNAi flowers and 35S:TM8 flowers had a split stamen cone (Fig. 4c, d). Only three flowers, one in each group, showed this in a severe form as described by [9] (Fig. 4c, Additional file 8). Extra floral organs as seen in Nicotiana were also observed, but again in very low numbers and both in control and in TM8-RNAi and 35S:TM8 lines (Fig. 4c, Additional file 8).

Together, we conclude that transgenic tomato plants overexpressing TM8 or silencing TM8 do not differ from controls and that no obvious function can be concluded for tomato. Our data suggest that TM8 does not act as a flowering repressor in Solanum lycopersium, as it does in Nicotiana.

\section{Expression profiles throughout evolution are diverse}

Although we cannot generalize a repressor function for TM8 to all Solanaceae, we asked if we could find a general pattern of expression throughout evolution. We compared available data from the literature to newly generated expression of TM8 in Cryptomeria japonica, Papaver somniferum, Vitis vinifera, Carica papaya and Antirrhinum majus (Fig. 5). Based on these data, we might cautiously say that throughout evolution expression in leaves decreased, while expression in all other tissues varies too much or not enough data is available yet to draw any conclusions. In general, these data support the idea that TM8 function is not widely conserved.

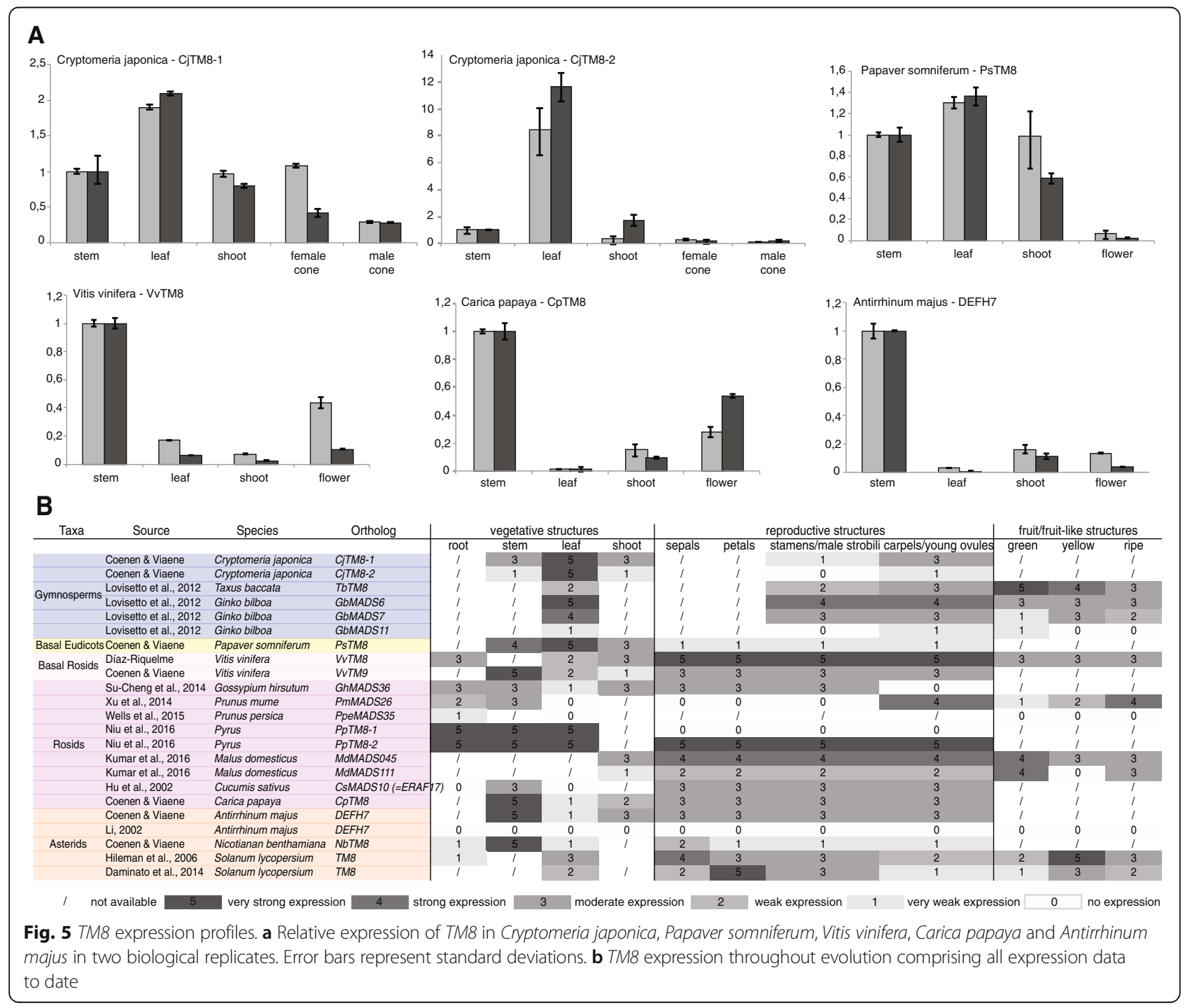




\section{Discussion}

While most MADS-box gene clades are well characterized in at least one model organism, only limited and often inconsistent findings have been published about the TM8-clade. In an effort to contribute new knowledge about TM8, we reconstructed its phylogenetic history in angiosperms and characterized the gene in three Solanaceae species.

The TM8-lineage is one of the more ancient MADS-box lineages as it was already present in the last common ancestor of the Spermatophytes [4]. We found that unlike most other MADS-box transcription factors, its duplicates were not retained after major duplication events like the $\varepsilon$ and $\gamma$ whole genome duplication and triplication, preceding the origin of the angiosperms and core eudicots respectively [33, 34]. In Solanaceae, which also underwent an additional whole genome triplication, TM8 is again mostly present as a single copy gene. The lack of duplicates in angiosperms is probably the main reason why TM8 was lost several independent times [6]. However, the fact that so many angiosperm species could flourish without it, suggests that its function became generally less necessary, that it became specific for certain environments or that its function rapidly evolved in angiosperms.

We have shown that in Nicotiana benthamiana the TM8 orthologue NbTM8 functions as a repressor of miR172. NbTM8 expression levels are positively regulated by NbSVP and their protein products interact. Both transcription factors have overlapping expression patterns in the shoot. Similar as for NbTM8, we identified $N b S V P$ to function as a repressor of $m i R 172$. While we are able to propose a function in Nicotiana benthamiana this function appears not to be strongly conserved in other closely related Solanaceae species. The lack of a clear phenotype in the Petunia TM8-mutant and both the TM8 overexpression and RNAi transgenic tomato lines, suggests that TM8 has no important function in these species. We must therefore conclude that TM8 function has strongly diverged or was even lost between these closely related species, even though their protein sequences appear to be conserved.

It appears that both SVP and TM8 are part of a regulatory module that rapidly evolved in Solanaceae species and may have broadly contributed to phenotypic diversity between species. Indeed, also SVP function diverged within Solanaceae. In the closely related Nicotiana tabacum NtSVP was found to regulate pedicel elongation [35]. In tomato the SVP homologue is JOINTLESS where it functions in abscission zone development and inflorescence architecture [36, 37]. In Physalis species MFP2 regulates the inflated calyx after pollination [31]. Outside of Solanaceae, the Antirrhinum majus homologue of SVP is INCOMPOSITA, which prevents prophyll development and controls floral meristem identity [38].

For tomato, our results do not fully correspond to those found in a similar study performed by Daminato et al. [9]. The authors described morphological malformations in the androecia in three out of 22 TM8 overexpression lines as the only macroscopic difference compared to wild-type tomatoes [9]. We observed the same phenotype together with other disturbances of the floral whorl patterning, both in OE, RNAi and control plants. It must be noted here that most flowers of all our T0-lines looked perfectly normal, and only a few flowers of a few plants displayed this anomaly, often in the first flowers to open. In Daminato et al., it was not specified how many flowers in the transgenic lines displayed this phenotype. Where normal wild-type tomatoes were used as control in Daminato et al., we used tomatoes obtained from uninoculated callus grown in vitro and we hypothesize that the malformations observed in both studies could possibly be due to somaclonal variation [39]. In addition, only two wild-type tomatoes were described as a control, and since the low prevalence of the phenotype, at least in our case, it is not unlikely that the authors therefore missed the presence of split stamen cones in wild-type lines in their study. Alternatively, the fact that we only generated two overexpression lines, may in turn explain why we didn't observe a consistent splayed out stamen phenotype.

Another explanation could be that Daminato et al. used the longer splice variant (NM_001247176.2) for their construct which indeed seems to occur regularly throughout Solanaceae as seen in our alignment (Additional file 6). We used the shorter variant as this was the clone we picked up from our cDNA. The short variant shows a deletion from bp 142 to bp 155 (located in the last part of the K-domain) and occurs regularly in Solanaceae. Thus far, nothing has been reported on the possible differences between both splice variants. It is not unlikely that the indel influences higher order complex formation, as is proposed for the K3-domain, and therefore the protein lost its functionality [40]. To evaluate the effect of the shorter and longer splice variant, both should be evaluated in the same experimental setup.

Finally, we noticed several intriguing similarities with another MADS-box gene, FLOWERING LOCUS C (FLC). $F L C$ plays a key role in the vernalization response in $A r a-$ bidopsis [41]. It represses flowering pathway integrators FLOWERING LOCUS $T$ (FT) and SUPPRESSOR OF OVEREXPRESSION OF CONSTANS1 (SOC1). It is epigenetically downregulated by cold, allowing the plant only to flower after a prolonged period of cold [42, 43]. A first similarity to TM8 is that both genes share the same location in the genome, as it was recently described that both 
are syntenic to SEPALATA3 (SEP3) [44, 45]. The FLC gene, however, originated later than $T M 8$, and it has been suggested that the $F L C$-like genes could be derived from a TM8 homolog in an ancestral angiosperm [44, 45]. Secondly, it is also notable that both genes are the MADS-box clades that were lost most frequently throughout angiosperms [6]. Interestingly, in most cases where one was lost, the other is still present, meaning that most genomes contain at least one of them. Thirdly, there seems to be a functional link as well: both have been assigned to be repressors of developmental transitions and flowering and act through regulation of the microRNA pathway, at least in Nicotiana regarding TM8 [46]. Furthermore, both share SVP as their interaction partner during these repressing activities [47]. Like $F L C$, the function of $S V P$ in the delay of the phase transitions is linked to temperature $[48,49]$. Considering that both FLC and SVP are temperature dependent genes, it seems an interesting option to evaluate the effect of temperature on TM8 functionality in future research.

\section{Conclusions}

We aimed to resolve one of the remaining open questions about MADS-box gene functions. We were able to generate functional data in three related Solanaceae species, but it remains difficult to generalize a function within angiosperms or even Solanaceae. While TM8 seems to perform a function as repressor of miR172 in Nicotiana benthamiana, its function seems to have diverged quickly between members of the same family. It has been proposed before that TM8 is a rapidly evolving gene [4] and our data are in line with this view.

\section{Methods}

All primers used throughout the research are listed in Additional file 9. All constructs were verified by sequencing before usage.

\section{Phylogenetic analyses}

Previously identified TM8 accessions from various species were used to blast (blastn) in all Viridiplantae genomes available to date provided by Phytozome 12.0 [20] and the Sol Genomics Network [50] (Additional file 1). All resulting coding sequences were aligned using MAFT and an initial phylogenetic tree was built using PhyML as implemented in Geneious with the GTR substitution model and default parameters [51, 52]. All sequences that fell within the strongly supported TM8-clade (SH-like branch support $=1$ ) were selected for further analysis. SOC1 genes were used as an outgroup to root the tree. The TM8-sequences detected in fully sequenced genomes were supplemented with hits resulting from blastn searches in the NCBI NT, EST and TSA databanks and the oneKP platform to find representatives in all angiosperm orders [21]. The final data matrix consisting of 177 sequences was again aligned by MAFT, manually optimized and the most likely tree was constructed using PhyML with SPR tree topology search and the GTR substitution model and evaluated by bootstrap analysis with 100 replicates [53]. Complete phylogeny including all accession numbers is provided in the Additional file 2.

\section{Plant material and growth conditions}

We received transgenic Nicotiana benthamiana lines overexpressing Arabidopsis miR-172a-1 from Xuemei Chen (UC Riverside, Mlotshwa et al. [30]). For Solanum lycopersicum the Micro-Tom cultivar was used in this study. For Petunia hybrida wild-type W138 and a W138-line with the 7 bp footprint of a $d T p h 1$ insertion in the PhTM8 start codon were used. All plants were grown at constant temperature $\left(25{ }^{\circ} \mathrm{C}\right)$ and long day conditions (16 h light, $8 \mathrm{~h}$ dark). $N$. benthamiana and Petunia seeds were first sown in Jiffy Pellets and grown in a conviron growth cabinet. Later, they were planted in pots and moved to a growth chamber.

\section{RNA isolation and CDNA preparation \\ RNA isolation and reverse transcription}

All sampled plant material was immediately frozen in liquid nitrogen and stored at $-80^{\circ} \mathrm{C}$. Total RNA was isolated using $\mathrm{TRIzol}^{\circ}$ following manufacturer's instructions (Invitrogen, Carlsbad, USA) and Dnase treated with TURBO DNA-free (Ambion, Austin, USA). RNA was reverse transcribed to cDNA using the GoScript reverse transcription system (Promega, Madison, USA). RNA quality was determined using the spectrophotometer, successful reverse transcription was tested by amplification of the ACTIN gene by PCR.

\section{RNA isolation for stem-loop qRT-PCR for microRNA quantification}

RNA was again isolated using TRIzol $^{\oplus}$ but for RNA precipitation we used twice the volume of isopropanol and precipitated for $30 \mathrm{~min}$ at $-80{ }^{\circ} \mathrm{C}$. cDNA was prepared using the AMV Reverse transcription kit (Promega, Wisconsin, U.S.A.). Following [54], we designed a custom stem-loop primer to specifically reverse transcribe microRNA172 and microRNA156. Reactions were performed in total RNA pools in the presence of an oligo-dT primer to reverse transcribe poly-A-tailed mRNA's using AMV reverse transcriptase (Promega, Madison, US).

\section{Characterization of NbTM8 and NbSVP in Nicotiana benthamiana} Cloning of NbTM8 and NbSVP, SOC1- and AP1-homologs

The full length sequence of NbTM8 was cloned from cDNA derived from floral buds based on the sequence 
of NtTM8 (EB449747). A partial sequence of SVP in N. benthamiana was present in the EST-database of Genbank (EH369950). We used 3'RACE to clone the 3'-sequence of NbSVP. A forward primer was combined with an oligodT to amplify the 3 'region. For APETALA1 (AP1), an alignment of homologs from Nicotiana tabacum (NAP1-2, AF009127) and Nicotiana sylvestris (NsMADS2, AF068726) was constructed. Forward and reverse primers were selected to amplify a 231-bp region of NbAP1. (SOC1) Based on SUPRESSOR OF CONSTANS1-homologs from Nicotiana tabacum (tobmads1, X76188) and Solanum lycopersicon (BG599624), a 339-bp fragment of NbSOC1 was amplified following a similar strategy. New sequences were added to GenBank.

\section{$q R T-P C R$ and developmental series}

To examine the expression of selected genes and microRNA's (NbTM8, NbSVP, NbSOC1, NbAP1, miR156 and miR172), qRT-PCR was used. Real-time PCR was performed on a StepOne Plus apparatus (Applied Biosystems, Forster City, US) using Fast SYBR Green Master Mix (Applied Biosystems, Forster City, US). Primers were designed using the Applied Biosystems Primer Express software. Quantification of mature microRNA levels was performed using primers complementary to the stem-loop primers in combination with microRNA forward complementary primers. To quantify genes or microRNA's in EV, VIGS or overexpression lines, inflorescence tissue of plants before they started to flower was used. All data presented here are three technical replicates from two biological replicates and are normalized against ACTIN expression. The developmental series was started from seedlings with two fully expanded cotyledons. A subsequent stage was sampled every time a new leaf had emerged. To measure expression in separate plant organs, whole organs of mature plants were sampled at anthesis of the first flower. In the developmental series and separate plant organs, pooled plant material was collected to obtain enough tissue for RNA isolation. Data were analyzed using the delta CT-method.

\section{In situ hybridization}

Sense and Antisense probes were in vitro transcribed using T7 RNA polymerase (New England Biolabs, Ipswich, AU) in the presence of digoxigenin-labeled UTP (Roche, Basel, $\mathrm{CH}$ ) from PCR amplified templates that included a T7 promoter. Tissues were fixed in $4 \%$ paraformaldehyde, paraffin embedded and sectioned at $8 \mu \mathrm{m}$. Sections were mounted on Probe-On-Plus slides (Fisher Scientific, Pittsburgh, US). Prehybridization, hybridization and detection were essentially following Carr \& Irish [55].

\section{Virus induced gene silencing of NbTM8 and NbSVP}

Gene-specific regions of NbTM 8 and NbSVP were introduced into the TRV2 vector [56]. The constructs were transformed into Agrobacterium strain GV3101 and used to infiltrate $N$. benthamiana. To quantify flowering time, $N$. benthamiana seeds were sown in long day conditions. A considerable number of plantlets (20-50) were infiltrated at the youngest possible stage (three to four leaf-stadium). After infiltration, plants were returned to their growth chamber and covered with foil for 2 days to keep them humid. Flowering time was counted as the number of leaves before the first flower appeared. As a control, plants were infiltrated with empty vector TRV2 and grown under the same conditions. Leaf and floral material from NbTM8-VIGS, NbSVP-VIGS plants showing phenotypes and empty vector control plants were collected in liquid nitrogen to check for effective downregulation.

\section{Overexpression of NbTM8 and NbSVP}

Full length sequences of $N b T M 8$ and $N b S V P$ from $N i$ cotiana benthamiana were cloned into a $35 \mathrm{~S}$ overexpression vector (pcB301, Filip Rolland). overexpression vectors were transformed into Agrobacterium strain GV3101. Stable transformation of Nicotiana benthamiana followed a transformation protocol optimized for tomato but using leaf-disks as explants [57]. Phenotypic characterization was performed in the T0 generation in comparison to T0 empty vector lines as strong 35S:NbTM8 lines were fully female sterile.

\section{Yeast two-hybrid}

Full length $N b T M 8$ and $N b S V P$ sequences were fused with the GAL4 activation domain in the pGAD424-vector and the GAL4 DNA-binding domain in the pGBT9 vector (Clontech, Mountain View, CA). Each possible vector combination was transformed in yeast strain Y187 as described in [58]. Two colonies per transformation were used for $\beta$-galactosidase liquid assays, and interaction was detected by use of ortho-Nitrophenyl- $\beta$-galactoside (ONPG) as a substrate [59]. Miller units as a quantification of $\beta$-galactosidase activity was calculated using the following formula: Miller units $=(1000 \times$ A420 $) /$ $(\mathrm{t} \times \mathrm{V} \times \mathrm{OD} 600)$ with $\mathrm{A} 420=$ absorbance at $420 \mathrm{~nm}$, OD600 $=$ Optical density at $600 \mathrm{~nm}, \mathrm{t}=$ number of minutes and $\mathrm{V}=0.5 \mathrm{~mL}$.

\section{Co-IP}

Full length NbTM 8 and NbSVP were cloned in the HBT95 expression vector [60] in frame with a double hemagglutinin (HA) or FLAG tag and subsequently maxi-prepped. Afterwards they were transformed in Arabidopsis protoplasts using PEG- $\mathrm{Ca}^{2}$ transformation and co-immunoprecipitated as described in [61]. Proteins were 
captured by agarose beads with a FLAG-antibody and co-immunoprecipitated proteins were visualized through Western Blot using HA-HRP and FLAG-HRP antibodies.

\section{Scanning electron microscopy}

The plant material was fixed in FAA (70\% ethanol:acetic acid:40\% formaldehyde, 90: 5: 5) and washed twice in $70 \%$ ethanol and dehydrated in a 1: 1 mixture of $70 \%$ ethanol and dimethoxymethan (DMM) for $5 \mathrm{~min}$ and in pure DMM for 20 min. After critical-point drying (CPD 030;BAL-TEC AG, Balzers, Liechtenstein), the dried material was mounted on aluminum stubs using Leit-C and coated with gold (SPI Module Sputter Coater; Spi Supplies, West Chester, PA, USA) before observation with a JEOL JSM-6360 SEM (Jeol Ltd., Tokyo, Japan).

\section{PhTM8 characterization in Petunia hybrida Identification of the phtm8 dTph1 insertion allele and derived footprint allele}

The phtm $8 d T p h 1$ insertion allele was identified by BLAST-searching a $d T p h 1$ transposon flanking sequence database [62], that has been considerably expanded in recent years. The presence of the $d T p h 1$ insertion was confirmed in planta in off-spring of the in silico identified insertion line, by PCR using a gene-specific primer pair flanking the insertion site. Among the different progenies, we identified several plants that were homozygous mutant for a putative footprint allele. Sequencing of the footprint allele showed a $7 \mathrm{bp}$ footprint insertion causing a frameshift mutation in the PhTM8 coding sequence. Progeny from homozygous WT and homozygous footprint mutants were obtained by selfing, and used for further phenotypic analysis.

\section{Phenotyping of the Petunia phtm8 footprint mutant}

Twenty-one W138 Petunia wild-types (WT) and 19 phtm8 footprint mutants were numbered and placed randomly together in the growth chambers. Plants were subsequently phenotyped for general anomalies and vegetative characteristics. 'Days to flowering' was counted from the day they were sown, till the day of full anthesis of the first flower. 'Number of leaves' include all true leaves (no cotyledons) before the first inflorescence. Upon flowering, flower characteristics like number of sepals, number of petals together with other anomalies of the first 20 flowers of each plant or until the end of the experiment were counted. Non-paired two-tailed t-tests were applied to determine if the means of the measured characteristics were significantly different between the control group and the footprint lines.

\section{Cloning of PhTM8}

Two PhTM8 primer-pairs were designed based on the predicted CDS of PhTM8 (received from M. VandenBussche).
In addition PhTM8 forward primers were combined with polyT reverse primers. PCR was carried out on cDNA from whole plant material of $6,8,12,14,16$, 18 and 20 leaves stadia, in inflorescence stadium and flowering stadium with annealing temperatures ranging between $47{ }^{\circ} \mathrm{C}$ and $60^{\circ}$ for $30-45$ cycles. All resulting amplicons of the proper length (around 579 bp) were cloned into the $\mathrm{pGEM}^{\circ}-\mathrm{T}$ Easy (Promega) and subsequently sequenced, but non matched with TM8. After our failed attempts to clone PhTM8, following datasets were blasted for PhTM8 expression: Petunia transcriptome [63], Unigenes and ESTs at the SOL Genomics Network (SGN) [50] and NCBI Transcriptome Shotgun Assembly (TSA) for Petunia species.

\section{Characterization of TM8 in Solanum lycopersicum Cloning of TM8}

Full-length tomato TM8 was cloned from mature leaf cDNA using primers based on the sequence (X60760.1) [7]. After confirmation by sequencing, it was cloned into pDONR 21 by BP clonase reaction and subsequently cloned into the $\mathrm{pK} 2 \mathrm{GW} 7$ vector by LR clonase reaction from Gateway Cloning [64]. For the RNAi-construct a $332 \mathrm{bp}$ long part of tomato TM8, starting from bp 266 in the I-domain and ending in the 3'UTR, was selected. The short TM8-fragment was blasted in the tomato genome to verify its specificity. No hits other than TM8 were found, proving that the selected sequence is highly specific and suited for RNAi. The 332 bp sequence was cloned into the pK7GWIWG2(I) vector. All destination vectors were confirmed by sequencing. The constructs were transformed into the LBA4404 Agrobacterium strain and selected on spec/strep/rif plates.

\section{Tomato transformations}

The tomato transformation protocol was followed as in [65]. Tomato cotyledons were cut in half and used as explants for Agro-infection. Transformed explants were further grown on 2Z-media supplemented with kanamycin (kan), allowing only successfully transformed explants to survive. Control lines were generated from non-transformed callus and grown on 2Z-medium without antibiotics. After shoot and root generation on MSSV + IBA + kan medium, transformed plants were planted in soil. All T0 and T1 plants were genotyped to confirm the presence of the constructs using a forward primer in the $35 \mathrm{~S}$ promotor and a reverse primer in the K-domain (Primer List). Semi-quantitative PCR confirmed much higher expression in $\mathrm{OE}$ lines compared to ctl, and no (or much lower) expression could be detected in silenced lines. Inflorescence with one open flower were sampled for the RT-PCR. 


\section{Phenotyping of tomato transformants}

All T0 plants were observed for abnormalities in vegetative or reproductive structures. Seed was harvested where possible and sown out again. T1 plants were subjected to elaborate phenotyping. Forty control, 40 RNAi and $36 \mathrm{OE}$ individuals were numbered and randomly located in the growth chamber. Plants were phenotyped in the same way as Petunia. The first three flowers of each plant were observed for number of petals, sepals, split stamen cones or other abnormalities. Statistical significance was determined by an unpaired two-tailed t-test.

\section{Additional files}

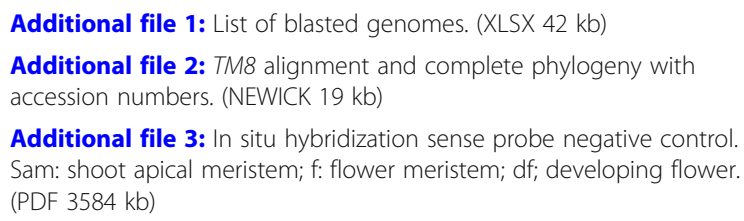

Additional file 4: Characterization of developmental phases in Nicotiana benthamiana. A) Juvenile-adult phase change in Nicotiana occurs around leaf 3-4 as indicated by the appearance of large trichomes on the leaf disk (indicated by black dots) and a pointed leaf tip. B) Detail of a large trichome indicative for the adult phase in Nicotiana. C) Trichome density increases faster in two Nicotiana transgenic lines overexpressing miR172 (L6 and L7). D) Relative expression of NbAP1 (left) and NbSOC1 (right) during Nicotiana development (cotyledons until the 14th leaf). The corresponding gray and black arrows indicate respectively floral transition and initiation of floral development. (PDF 11213 kb)

Additional file 5: Illustration of footprint mutant sequence. Wild-type PhTM8 sequence around the start codon compared to the phtm 8 sequence around the new startcodon created by a dTph1 transposon insertion. The dTph1 transposable element left a 7 bp footprint (underlined) creating a new start codon (red) which leads to an immediate frameshift PhTM8. The newly translated protein of the footprint mutant results quickly in a stop codon leading to a short non-sense protein. (PNG $21 \mathrm{~kb}$ )

Additional file 6: TM8 protein alignment of Solanaceae reveals different splice variants. PhTM8 aligns well to its orthologs and does not show any sign of pseudogenization. (PDF $1315 \mathrm{~kb}$ )

Additional file 7: RT-PCR of TM8 and ACTIN in tomato in T1phenotyped lines. Control, TM8 overexpression (OE) and TM8-RNAi lines (31 cycles all starting from $10 \mathrm{ng} / \mu \mathrm{L}$ cDNA obtained from inflorescences with at least one open flower). (PDF $46 \mathrm{~kb}$ )

Additional file 8: Abnormalities observed in callus cultivated tomato plants. A) normal wild-type flower consisting of five petals, five sepals, a cone formed by five stamens and an ovary within. B) Control flower with interrupted and sepaloïd stamen cone. C) RNAi flower with extra floral organs and crown anthers. D) OE flower with sepaloïd petals, E) split stamen cone, F) fusion of stamen and pistil, and G) disturbed growth and leaf morphology. H) RNAi flower with splayed out stamen cone I) OE flower with sepaloïd stamen. In picture E) and F) petals and sepals were removed for better sight on stamens and pistil. (PDF $202 \mathrm{~kb}$ )

Additional file 9: Primer list. (XLSX $43 \mathrm{~kb}$ )

\section{Abbreviations}

AP1: APETALA1; AP2: APETALA2; FLC: FLOWERING LOCUS C; FT: FLOWERING LOCUS T; OE: Overexpression; SOC1: Suppressor of constans 1; SVP: Short vegetative phase; TM8: Tomato MADS 8; VIGS: Virus induced gene silencing

\section{Acknowledgements}

We would like to thank Hanne Mariën and Matthew Ramon for performing the Co-IP experiment and Filip Rolland for providing us the pcB301 vector.
We thank Ellen De Sutter for technical assistance in preparing the transgenic tomatoes. Furthermore, we kindly thank Patrice Morel for assistance with genotyping in Petunia. Finally we would like to thank Xuemei Chen (UC Riverside) for the transgenic miR172 overexpression lines.

\section{Funding}

HC was funded by KU Leuven. TV was funded by a scholarship from IWT Vlaanderen (no. 35040) and a PDM scholarship from KU Leuven. MV was supported by a CNRS ATIP-AVENIR award. KG was funded by KULeuven grants C24/17/037 and OT/12/053 and FWO grant G065713 N.

\section{Availability of data and materials}

All data generated or analysed during this study are included in this published article and its supplementary information files.

\section{Authors' contributions}

TV generated and analyzed all Nicotiana data, all expression data in Cryptomeria japonica, Papaver somniferum, Vitis vinifera, Carica papaya and Antirrhinum majus and wrote parts of the draft manuscript. HC performed the phylogenetic analyses, generated and analysed all Petunia and tomato data and wrote the manuscript. MV identified the phtm8 mutant and predicted the PhTM8 CDS. KG designed and coördinated the experiments, analyzed the data and wrote the manuscript. All authors read and approved the final version of the manuscript.

Ethics approval and consent to participate

Not applicable.

\section{Consent for publication}

Not applicable.

\section{Competing interests}

The authors declare that they have no competing interests.

\section{Publisher's Note}

Springer Nature remains neutral with regard to jurisdictional claims in published maps and institutional affiliations.

\section{Author details}

'Department of Biology, KU Leuven, Kasteelpark Arenberg 31, B-3001 Leuven, Belgium. 'Laboratoire Reproduction et Développement des Plantes,

University Lyon, ENS de Lyon, UCB Lyon 1, CNRS, INRA, F-69342 Lyon, France.

Received: 31 October 2017 Accepted: 11 June 2018

Published online: 22 June 2018

\section{References}

1. Theissen G, Becker A, Di Rosa A, Kanno A, Kim JT, Münster T, et al. A short history of MADS-box genes in plants. In: Plant Molecular Evolution; 2000. p. 115-49.

2. Becker A, Theissen G. The major clades of MADS-box genes and their role in the development and evolution of flowering plants. Mol Phylogenet Evol. 2003;29:464-89.

3. Smaczniak C, Immink RGH, Angenent GC, Kaufmann K. Developmental and evolutionary diversity of plant MADS-domain factors: insights from recent studies. Development. 2012;139:3081-98.

4. Gramzow L, Weilandt L, Theißen G. MADS goes genomic in conifers: towards determining the ancestral set of MADS-box genes in seed plants. Ann Bot. 2014;114:1407-29.

5. Heijmans K, Morel P, Vandenbussche M. MADS-box genes and floral development: the dark side. J Exp Bot. 2012;63:5397-404.

6. Gramzow L, Günter T. Phylogenomics reveals surprising sets of essential and dispensable clades of MIKC c -group MADS-box genes in flowering plants. J Exp Zool B Mol Dev Evol. 2015;324:353-62.

7. Pnueli L, Abu-Abeid M, Zamir D, Nacken W, Schwarz-Sommer Z, Lifschitz E. The MADS box gene family in tomato: temporal expression during floral development, conserved secondary structures and homology with homeotic genes from Antirrhinum and Arabidopsis. Plant J. 1991;1:255-66.

8. Lifschitz E, Brodai L, Hareven D, Hurwitz C, Prihadash A, Pnueli L, et al. Molecular mapping of flower development in tomato. In: Yoder J, editor. 
Molecular Biology of Tomato. Pennsylvania: Technomic Publishing Company; 1993. p. 175-84

9. Daminato M, Masiero S, Resentini F, Lovisetto A, Casadoro G. Characterization of TM8, a MADS-box gene expressed in tomato flowers. BMC Plant Biol. 2014;14:319.

10. Ando S, Sato Y, Kamachi S, Sakai S. Isolation of a MADS-box gene (ERAF17) and correlation of its expression with the induction of formation of female flowers by ethylene in cucumber plants (Cucumis sativus L). Planta. 2001;213:943-52.

11. Mingai LI. Molecular and genetic characterization of new MADS-box genes in antirrhinum majus. PhD Thesis. Universität zu Köln. 2002.

12. Hileman LC, Sundstrom JF, Litt A, Chen M, Shumba T, Irish VF. Molecular and Phylogenetic Analyses of the MADS-Box Gene Family in Tomato. Mol Biol Evol. 2006;23:2245-58.

13. Díaz-Riquelme J, Lijavetzky D, Martínez-Zapater JM, Carmona MJ. Genomewide analysis of MIKCC-type MADS box genes in grapevine. Plant Physiol. 2009;149:354-69.

14. Díaz-Riquelme J, Martínez-Zapater JM, Carmona MJ. Transcriptional analysis of tendril and inflorescence development in grapevine (Vitis vinifera L.). PLoS One. 2014;9:e92339

15. Lovisetto A, Guzzo F, Tadiello A, Toffali K, Favretto A, Casadoro G. Molecular analyses of MADS-box genes trace back to Gymnosperms the invention of fleshy fruits. Mol Biol Evol. 2011;29:409-19.

16. Xu Z, Zhang Q, Sun L, Du D, Cheng T, Pan H, et al. Genome-wide identification, characterisation and expression analysis of the MADS-box gene family in Prunus mume. Mol Gen Genomics. 2014;289:903-20.

17. Wells CE, Vendramin E, Jimenez Tarodo S, Verde I, Bielenberg DG. A genome-wide analysis of MADS-box genes in peach [Prunus persica $(\mathrm{L}$.) Batsch]. BMC Plant Biol. 2015;15:41.

18. Niu Q, Li J, Cai D, Qian M, Jia H, Bai S, et al. Dormancy-associated MADS-box genes and microRNAs jointly control dormancy transition in pear (Pyrus pyrifolia white pear group) flower bud. J Exp Bot. 2016;67:239-57.

19. Kumar G, Arya P, Gupta K, Randhawa V, Acharya V, Singh AK. Comparative phylogenetic analysis and transcriptional profiling of MADS-box gene family identified DAM and FLC-like genes in apple (Malusx domestica). Sci Rep. 2016:6:20695.

20. Goodstein DM, Shu S, Howson R, Neupane R, Hayes RD, Fazo J, et al. Phytozome: a comparative platform for green plant genomics. Nucleic Acids Res. 2012;40:D1178-86.

21. Matasci N, Hung L-H, Yan Z, Carpenter EJ, Wickett NJ, Mirarab S, et al. Data access for the 1,000 Plants (1KP) project. Gigascience. 2014;3:17.

22. Maere S, De Bodt S, Raes J, Casneuf T, Van Montagu M, Kuiper M, et al. Modeling gene and genome duplications in eukaryotes. Proc Natl Acad Sci. 2005;102:5454-9.

23. Theissen G, Kim JT, Saedler H. Classification and phylogeny of the MADSbox multigene family suggest defined roles of MADS-box gene subfamilies in the morphological evolution of eukaryotes. J Mol Evol. 1996;43:484-516.

24. Geuten K, Viaene T, Vekemans D, Kourmpetli S, Drea S. Analysis of developmental control genes using virus-induced gene silencing. Methods Mol Biol. 2013;975:61-9.

25. Telfer A, Bollman KM, Poethig RS. Phase change and the regulation of trichome distribution in Arabidopsis thaliana. Development. 1997;124:645-54.

26. Samach A, Onouchi H, Gold SE, Ditta GS, Schwarz-Sommer Z, Yanofsky MF, et al. Distinct Roles of CONSTANS Target Genes in Reproductive Development of Arabidopsis. Science. 2000;288:1613-6.

27. Cho HJ, Kim J, Lee JH, Kim W, Jung J-H, Park C-M, et al. SHORT VEGETATIVE PHASE (SVP) protein negatively regulates miR172 transcription via direct binding to the pri-miR172a promoter in Arabidopsis. FEBS Lett. 2012;586:2332-7.

28. Hartmann U, Höhmann S, Nettesheim K, Wisman E, Saedler H, Huijser P. Molecular cloning of SVP: a negative regulator of the floral transition in Arabidopsis. Plant J. 2000;21:351-60.

29. Gregis V, Sessa A, Colombo L, Kater MM. AGL24, SHORT VEGETATIVE PHASE, and APETALA1 redundantly control AGAMOUS during early stages of flower development in Arabidopsis. Plant Cell. 2006;18:1373-82.

30. Mlotshwa S, Yang Z, Kim Y, Chen X. Floral patterning defects induced by Arabidopsis APETALA2 and microRNA172 expression in Nicotiana benthamiana. Plant Mol Biol. 2006;61:781-93.

31. He C, Saedler H. Heterotopic expression of MPF2 is the key to the evolution of the Chinese lantern of Physalis, a morphological novelty in Solanaceae. Proc Natl Acad Sci. 2005:102:5779-84.

32. Immink RGH, Ferrario S, Busscher-Lange J, Kooiker M, Busscher M, Angenent GC. Analysis of the petunia MADS-box transcription factor family. Mol Gen Genomics. 2003;268:598-606
33. Jiao Y, Wickett NJ, Ayyampalayam S, Chanderbali AS, Landherr L, Ralph PE, et al. Ancestral polyploidy in seed plants and angiosperms. Nature. 2011; 473:97-100

34. Vekemans D, Proost $\mathrm{S}$, Vanneste $\mathrm{K}$, Coenen $\mathrm{H}$, Viaene T, Ruelens $\mathrm{P}$, et al. Gamma Paleohexaploidy in the Stem Lineage of Core Eudicots: Significance for MADS-Box Gene and Species Diversification. Mol Biol Evol. 2012;29:3793806.

35. Wang $D$, Chen X, Zhang Z, Liu D, Song G, Kong X, et al. A MADS-box gene NtSVP regulates pedicel elongation by directly suppressing a KNAT1-like KNOX gene NtBPL in tobacco (Nicotiana tabacum L.). J Exp Bot. 2015;66: 6233-44.

36. Szymkowiak EJ, Irish EE. JOINTLESS suppresses sympodial identity in inflorescence meristems of tomato. Planta. 2006;223:646-58.

37. Liu D, Wang D, Qin Z, Zhang D, Yin L, Wu L, et al. The SEPALLATA MADSbox protein SLMBP21 forms protein complexes with JOINTLESS and MACROCALYX as a transcription activator for development of the tomato flower abscission zone. Plant J. 2014;77:284-96.

38. Masiero S, Li M-A, Will I, Hartmann U, Saedler H, Huijser P, et al. INCOMPOSITA: a MADS-box gene controlling prophyll development and floral meristem identity in Antirrhinum. Development. 2004;131:5981-90.

39. Khaliluev MR, Chaban IA, Kononenko NV, Baranova EN, Dolgov SV, Kharchenko PN, et al. Abnormal floral meristem development in transgenic tomato plants do not depend on the expression of genes encoding defense-related PR-proteins and antimicrobial peptides. Russ J Dev Biol. 2014:45:22-33.

40. Kaufmann K, Melzer R, Theissen G. MIKC-type MADS-domain proteins: structural modularity, protein interactions and network evolution in land plants. Gene. 2005;347:183-98.

41. Michaels SD, Amasino RM. FLOWERING LOCUS C Encodes a Novel MADS Domain Protein That Acts as a Repressor of Flowering. Plant Cell. 1999;1 1:949.

42. Bastow R, Mylne JS, Lister C, Lippman Z, Martienssen RA, Dean C. Vernalization requires epigenetic silencing of $\mathrm{FLC}$ by histone methylation. Nature. 2004;427:164-7.

43. Michaels SD, Himelblau E, Kim SY, Schomburg FM, Amasino RM. Integration of flowering signals in winter-annual Arabidopsis. Plant Physiol. 2005;137:149-56.

44. Zhao T, Holmer R, Bruijn S de, Angenent GC, van den Burg HA, Schranz ME. Phylogenomic Synteny Network Analysis of MADS-Box Transcription Factor Genes Reveals Lineage-Specific Transpositions, Ancient Tandem Duplications, and Deep Positional Conservation. Plant Cell. 2017;29:1278-92.

45. Ruelens P, de Maagd RA, Proost S, Theißen G, Geuten K, Kaufmann K. FLOWERING LOCUS $C$ in monocots and the tandem origin of angiospermspecific MADS-box genes. Nat Commun. 2013;4:2280.

46. Deng W, Ying H, Helliwell CA, Taylor JM, Peacock WJ, Dennis ES. FLOWERING LOCUS C (FLC) regulates development pathways throughout the life cycle of Arabidopsis. Proc Natl Acad Sci U S A. 2011;108:6680-5.

47. Li D, Liu C, Shen L, Wu Y, Chen H, Robertson M, et al. A repressor complex governs the integration of flowering signals in Arabidopsis. Dev Cell. 2008;15:110-20

48. Lee JH, Ryu H-S, Chung KS, Pose D, Kim S, Schmid M, et al. Regulation of Temperature-Responsive Flowering by MADS-Box Transcription Factor Repressors. Science. 2013;342:628-32.

49. Posé $\mathrm{D}$, Verhage L, Ott F, Yant L, Mathieu J, Angenent GC, et al. Temperature-dependent regulation of flowering by antagonistic FLM variants. Nature. 2013;503:414-7.

50. Bombarely A, Menda N, Tecle IY, Buels RM, Strickler S, Fischer-York T, et al. The Sol Genomics Network (solgenomics.net): growing tomatoes using Perl. Nucleic Acids Res. 2011;39:D1149-55.

51. Guindon S, Dufayard J-F, Lefort V, Anisimova M, Hordijk W, Gascuel O. New Algorithms and Methods to Estimate Maximum-Likelihood Phylogenies: Assessing the Performance of PhyML 3.0. Syst Biol. 2010;59:307-21.

52. Kearse M, Moir R, Wilson A, Stones-Havas $S$, Cheung M, Sturrock $S$, et al. Geneious Basic: an integrated and extendable desktop software platform for the organization and analysis of sequence data. Bioinformatics. 2012;28:1647-9.

53. Felsenstein J. Confidence limits on phylogenies: an approach using the bootstrap. Evolution. 1985;39:783-91.

54. Chen C, Ridzon DA, Broomer AJ, Zhou Z, Lee DH, Nquyen JT, et al. Realtime quantification of microRNAs by stem-loop RT-PCR. Nucleic Acids Res. 2005;33:e179.

55. Carr SM, Irish VF. Floral homeotic gene expression defines developmental arrest stages inBrassica oleracea L. vars.botrytis anditalica. Planta. 1997;201: 179-88. 
56. Dinesh-Kumar SP, Anandalakshmi R, Marathe R, Schiff M, Liu Y. Virusinduced gene silencing. Methods Mol Biol. 2003;236:287-94.

57. Geuten K, Irish V. Hidden variability of floral homeotic B genes in Solanaceae provides a molecular basis for the evolution of novel functions. Plant Cell. 2010;22:2562-78.

58. Gietz RD, Woods RA. Yeast transformation by the LiAc/SS Carrier DNA/PEG method. Methods Mol Biol. 2006;313:107-20

59. Miller JH. Experiments in molecular genetics. New York: Cold Spring Harbor Laboratory Pr; 1972.

60. Sheen J. Ca2+-dependent protein kinases and stress signal transduction in plants. Science. 1996;274:1900-2.

61. Ramon M, Ruelens P, Li Y, Sheen J, Geuten K, Rolland F. The hybrid fourCBS-domain KIN $\beta y$ subunit functions as the canonical $y$ subunit of the plant energy sensor SnRK1. Plant J. 2013;75:11-25.

62. Vandenbussche M, Janssen A, Zethof J, van Orsouw N, Peters J, van Eijk MJT, et al. Generation of a 3D indexed Petunia insertion database for reverse genetics. Plant J. 2008;54:1105-14.

63. Villarino GH, Bombarely A, Giovannoni JJ, Scanlon MJ, Mattson NS. Transcriptomic analysis of Petunia hybrida in response to salt stress using high throughput RNA sequencing. PLoS One. 2014;9:e94651.

64. Karimi M, Inzé D, Depicker A. GATEWAYTM vectors for Agrobacteriummediated plant transformation. Trends Plant Sci. 2002;7:193-5.

65. McCormick S. Transformation of tomato with Agrobacterium tumefaciens. In: Plant Tissue Culture Manual; 1991. p. 311-9.

\section{Ready to submit your research? Choose BMC and benefit from:}

- fast, convenient online submission

- thorough peer review by experienced researchers in your field

- rapid publication on acceptance

- support for research data, including large and complex data types

- gold Open Access which fosters wider collaboration and increased citations

- maximum visibility for your research: over $100 \mathrm{M}$ website views per year

At BMC, research is always in progress.

Learn more biomedcentral.com/submissions 